\title{
Energy spectrum of the 3D velocity field, induced by vortex tangle
}

XX.XX.2007

Keywords superfluidity, vortices, quantum turbulence

Abstract A review of various exactly solvable models on the determination of the energy spectra $E(k)$ of $3 \mathrm{D}$-velocity field, induced by chaotic vortex lines is proposed. This problem is closely related to the sacramental question whether a chaotic set of vortex filaments can mimic the real hydrodynamic turbulence. The quantity $\langle\mathbf{v}(\mathbf{k}) \mathbf{v}(-\mathbf{k})>$ can be exactly calculated, provided that we know the probability distribution functional $\mathscr{P}(\{\mathbf{s}(\xi, t)\})$ of vortex loops configurations. The knowledge of $\mathscr{P}(\{\mathbf{s}(\xi, t)\})$ is identical to the full solution of the problem of quantum turbulence and, in general, $\mathscr{P}$ is unknown. In the paper we discuss several models allowing to evaluate spectra in the explicit form. This cases include standard vortex configurations such as a straight line, vortex array and ring. Independent chaotic loops of various fractal dimension as well as interacting loops in the thermodynamic equilibrium also permit an analytical solution. We also describe the method of an obtaining the 3D velocity spectrum induced by the straight line perturbed with chaotic $1 \mathrm{D}$ Kelvin waves on it.

PACS number(s): 67.25.dk, 47.37.+q, 03.75.Kk

\section{Introduction}

One of the exciting applications of quantum turbulence is the solution (or rather, the attempt at a solution) of the tantalizing problem of classical turbulence. For this reason, the problem of the spectrum of the 3D velocity field induced by a chaotic vortex filament becomes one of the central questions. The formal relation, allowing of calculating $E(\mathbf{k})=\rho_{s}\left\langle\mathbf{v}_{\mathbf{k}} \mathbf{v}_{-\mathbf{k}}\right\rangle$ via the vortex line configuration

Institute of Thermophysics

Novosibirsk, 630090, Russia

E-mail: nemir@itp.nsc.ru 
$\{\mathbf{s}(\xi)\}$, is given by formula (see $-\mathbf{1})$

$$
E=\left\langle\frac{\rho_{s} \kappa^{2}}{2} \int_{\mathbf{k}} \frac{d^{3} \mathbf{k}}{\mathbf{k}^{2}} \int_{0}^{L} \int_{0}^{L} \mathbf{s}^{\prime}\left(\xi_{1}\right) \mathbf{s}^{\prime}\left(\xi_{2}\right) d \xi_{1} d \xi_{2} \exp \left[i \int_{\xi_{1}}^{\xi_{2}} \mathbf{k s}^{\prime}(\tilde{\xi}) d \tilde{\xi}\right]\right\rangle .
$$

In isotropic case, the spectral density depends on the absolute value of the wave number $k$. Integrating over solid angle leads to formula ( $\sec ^{2}$ ):

$$
E(k)=\left\langle\frac{\rho_{s} \kappa^{2}}{(2 \pi)^{2}} \int_{0}^{L} \int_{0}^{L} \mathbf{s}^{\prime}\left(\xi_{1}\right) \mathbf{s}^{\prime}\left(\xi_{2}\right) d \xi_{1} d \xi_{2} \frac{\sin \left(k\left|\mathbf{s}\left(\xi_{1}\right)-\mathbf{s}\left(\xi_{2}\right)\right|\right)}{k\left|\mathbf{s}\left(\xi_{1}\right)-\mathbf{s}\left(\xi_{2}\right)\right|}\right\rangle .
$$

For anisotropic situations, formula (2) is understood as an angle average. Further we will apply these formulas to study some particular situation.

Of course, the relations (2)-(1) are just mathematical identities, and the physics is hidden behind the \langle\rangle operation. To calculate this average, we need the probability distribution functional $\mathscr{P}(\{\mathbf{s}(\xi)\}, t)$, which is the probability that the system has the configuration $\{\mathbf{s}(\xi)\}$ of the set of the vortex loops. Knowing $\mathscr{P}(\{\mathbf{s}(\xi)\}, t)$ is identical to the full solution of the problem of quantum turbulence. Therefore, the problem of the determination of the 3D energy spectrum is not resolved, although there are a series of theoretical approaches and numerical simulations. We consider here several exactly solvable cases, the study of them is quite instructive.

\section{Regular structures (straight line, vortex array, ring)}

The angle averaged spectrum created by straight vortex line (directed along axis $z$ ) is directly evaluated from (2)

$$
\int_{\mathbf{k}} d k \frac{\rho_{s} \kappa^{2}}{(2 \pi)^{2}}\left\langle\int_{0}^{L} \int_{0}^{L} \frac{\sin \left(k\left(z_{1}-z_{2}\right)\right)}{\left(k\left(z_{1}-z_{2}\right)\right)} d z_{1} d z_{2}\right\rangle=\frac{\rho_{s} \kappa^{2} L}{4 \pi} \int_{\mathbf{k}} d k \frac{1}{k} .
$$

The length $L$ is the conditional quantity. more correctly to talk here about energy per unit length. Spectrum of the straight line (3) is discussed early (see, e.g., $\frac{3}{,}, \frac{4}{,}$ ), Vinen $\frac{5}{5}$ proposed the $k^{-1}$ spectrum on the basis of dimensional consideration. This result is important, since it (3) states that for any vortex system the high wave numbers larger than inverse curvature, $E(k)$ should be $\left(\rho_{s} \kappa^{2} / 4 \pi\right) k^{-1}$ (per unit length).

Let us take a set of straight vortex filaments forming the square lattice $\bigcup s_{i}(\xi)=$ $\mathrm{U}\left(x_{i}, y_{i}, z\right)$. Points $x_{i}, y_{i}$ are coordinates for vortices on the $x y$-plane, index $i$ runs from 1 to $N$. The neighboring lines are separated by distance $b$, i.e., $x_{i+1}-x_{i}=b$. Then the general relation (3) leads to the following formula (Hanninen (private communication), Nowak et al. $(\underline{6})$ :

$$
\frac{E(k)}{\rho_{s} \kappa^{2} L}=\frac{1}{4 \pi k} \sum_{i=1}^{N} \sum_{i=1}^{N} J_{0}\left(k d_{i j}\right)
$$


where $d_{i j}=\sqrt{\left(x_{i}-x_{j}\right)^{2}+\left(y_{i}-y_{j}\right)^{2}}$ distances between vortices on the $x y$-plane. Thus, determination of the spectrum on the basis (4) should be done with the use of the quadruple summation (over $\left(x_{i}, x_{j}, y_{i}, y_{j}\right)$ ), which requires large computing resources. Clear, however, that for very small $k$, which corresponds to very large distance, the whole array can be considered as large single vortex with the circulation $N^{2} \kappa$. Accordingly, the spectrum (per unit height) should be $\left(\rho_{s} N^{4} \kappa^{2} / 4 \pi\right) k^{-1}$. For large $k$, which corresponds to very small distance from each line, the spectrum (per unit height) should be $\left(\rho_{s} \kappa^{2} / 4 \pi\right) k^{-1}$ In the intermediate region $k b<<1$, and $N k b>>1$ (this condition implies that inverse wave number $k^{-1}$ is larger intervortex space between neighboring lines, but smaller then the size of the whole array $N d_{i j}$ ), we can replace the quadruple summation by the quadruple integration with infinite limits. This procedure corresponds that we exclude the fine-scale motion near each of vortex, and are interested in the only large-scale, coarse-grained motion. After obvious change of variables $x_{i} \rightarrow k x_{i}, y_{i} \rightarrow k y_{i}$ etc. we get that the the whole integral should scale as $1 / k^{4}$, and accordingly

$$
E(k) \propto 1 / k^{5}
$$

Formula $E(k)=d E / d k \propto 1 / k^{5}$ implies that $E(\mathbf{k})=d E / d^{2} k$ should behave as $1 / k^{-6}$. It follows, for example, from that in the $2 \mathrm{D}$ case $d^{2} k=2 \pi k d k$ (isotropic is assumed). Since, further, $E(\mathbf{k})=v(\mathbf{k}) v(-\mathbf{k})$, we see that $v(\mathbf{k})$ scales as $1 / k^{-3}$. The latter means (see, e.g.,,$\underline{7}$, Eqs. (4.60),(4.61)) that the velocity $v(\mathbf{r})$ scales as $\mathbf{r}^{1}$. Thus, the uniform vortex array creates the course-grained motion, which is rotation, as it should be. Moreover, the coefficient is equal to $\kappa / 2 b^{2}$, which coincides with the Feynman rule.

Now we will consider a vortex ring with radius $R$ lying in the $x-y$ plane. The line $s(\xi)$ can be parameterized as $\mathbf{s}(\mathbf{x})=(R \cos \varphi, R \sin \varphi, 0)$ with $\varphi \in[0,2 \pi]$. Applying it to Eq. (2) we get (see also $\underline{\text { ) }}$ )

$$
E_{\text {ring }}=\frac{\rho_{s} \kappa^{2} R}{(2 \pi)^{2}} \int_{0}^{2 \pi} \int_{0}^{2 \pi} d \varphi_{1} d \varphi_{2} \frac{\cos \left(\varphi_{1}-\varphi_{2}\right) \sin \left(2 k R \sin \left(\left(\varphi_{1}-\varphi_{2}\right) / 2\right)\right)}{2 k \sin \left(\left(\varphi_{1}-\varphi_{2}\right) / 2\right)}
$$

Evaluating the integral numerically shows that the spectrum $E_{\text {ring }}(k)$ scales like $E_{\text {ring }}(k) \sim k^{2}$ for $k R \ll 1$. Frequently, the $k^{2}$ is referred to as a proof for the thermodynamical equilibrium state. We would like to stress, however, that this distribution of the energy (valid far from the ring) has nothing to do with the equipartition law. It is a consequence of the fact that closed vortex loops induce a far field flow scaling as $1 / r^{3}$. That, in turn, generates a spectrum $E(k) \propto k^{2}$. This fact was established for classical turbulence (see, e.g. $\frac{8}{\stackrel{8}{*}}$ ). For quantum turbulence this result was discussed by Stalp, Skrbek and Donnelly ${ }^{9}$. For the large $k$, namely for $k R \gg 1$, spectrum $E_{\text {ring }}(k)$ scales like $\sim k^{-1}$ as for straight line.

\section{Noninteracting Gaussian loops of various fractal dimension}

One of the approaches, allowing exact solution, based on the viewing of the VT as a set of loops having the random structure with the various fractal dimension 10 . 

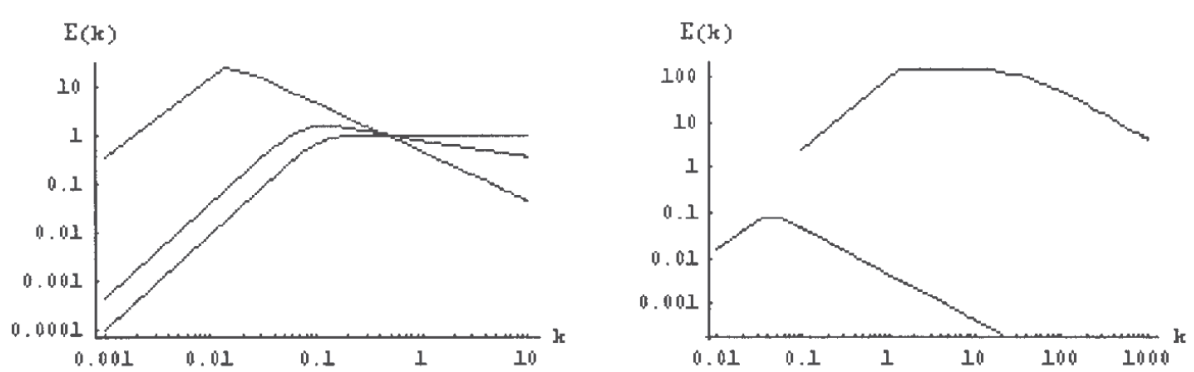

Fig. 1 (Color online) Energy spectra of a pure fractal line (left), and a more realistic semi-fractal vortex loop (right).

This theory is based on the Gaussian model (see $\left.\frac{1}{2}\right)$. The probability $\mathscr{P}_{\text {Gauss }}(\{\mathbf{s}(\xi, t)\})$ of finding a particular configuration $\{\mathbf{s}(\xi, t)\}$ is expressed by the probability distribution functional (for details, see the paper by author $\frac{1}{\underline{1}}$ )

$$
\mathscr{P}_{\text {Gauss }}(\{\mathbf{s}(\xi, t)\})=\mathscr{N} \exp \left(-\int_{0}^{l} \int_{0}^{l} \mathbf{s}^{\prime \alpha}\left(\xi_{1}, t\right) \Lambda_{\alpha \beta}\left(\xi_{1}-\xi_{2}\right) \mathbf{s}^{\prime \beta}\left(\xi_{2}, t\right) d \xi_{1} d \xi_{2}\right) .
$$

Here $\mathscr{N}$ is a normalizing factor and $l$ is the length of curve. The typical form of function $\Lambda_{\alpha \beta}\left(\xi-\xi^{\prime}\right)$ is a smoothed $\delta$ function, having a Mexican hat shape with width equal to $\xi_{0}$. Behavior of $\Lambda_{\alpha \beta}\left(\xi_{1}-\xi_{2}\right)$ on large scales determines the second order correlation function between tangent vectors $\left\langle\mathbf{s}_{\alpha}^{\prime}\left(\xi_{1}\right) \mathbf{s}_{\alpha}^{\prime}\left(\xi_{2}\right)\right\rangle$, which, in turn, determines the fractal dimension of line. Referring the reader to the original article $\frac{10}{}$ for the details we briefly state the main results.

In the region $k \ll 1 / L^{1 / H_{D}}$, the spectrum behaves as $E(k) \propto k^{2}$. We again stress that this dependence is related to the far field flow $1 / r^{3}$ from the restricted domain of vorticity (but not to the thermodynamical equilibrium). Note, that $L^{1 / H_{D}}$ is nothing but the real 3D size of the loop. In the opposite case, of large $k$ we get

$$
E(k) \propto k^{-2+H_{D}} .
$$

This result had been obtained earlier from qualitative considerations and is discussed in Frisch ${ }^{7}$. Fig. 1 (left) depicts (in logarithmic scale) three curves, the spectral densities $E(k)$ for a pure fractal vortex filament of length $L=100$ (all units are arbitrary) and three different values of $H_{D}=1,5 / 3,2$ correspondingly. It can be seen how $E(k) \propto k^{2}$ is changed to a dependence $E(k) \propto k^{-2+H_{D}}$ in the region of large $k \sim 1 / 100^{-1 / H_{D}}$.

Vortex loops in superfluid turbulent quantum fluids are "semifractal" objects, which are smooth at small $\left(\xi^{\prime}-\xi^{\prime \prime}\right)$ along the curve, and are fully uncorrelated for the remote parts. A good approximation for $\left\langle\mathbf{s}_{\alpha}^{\prime}\left(\xi_{1}\right) \mathbf{s}_{\alpha}^{\prime}\left(\xi_{2}\right)\right\rangle$ is a function of the type $1 /\left(1+\left(\Delta \xi / \xi_{0}\right)^{2}\right)$.

Fig. 1 (right) shows (in logarithmic scale) the spectral densities $E(k)$ for a "semifractal" vortex loop of length $L=100$ with $\xi_{0}=0.01$ and $\xi_{0}=1$. It is clearly seen that the curves $\left(\xi_{0}=0.01\right)$ have three regions with bends at the points $k_{l}=$ $1 / \sqrt{L \xi_{0}}=1$, and $k_{r}=1 / \xi_{0}=100$. In these three different regions we have $E(k) \propto$ 
$k^{2}, E(k) \propto k^{0}$ and $E(k) \propto k^{-1}$, correspondingly. This is in excellent agreement with the qualitative considerations.

\section{Thermodynamically equilibrium vortex tangle}

In the previous Section, we considered the model of free (noninteracting) vortex loops. Let us now discuss another solvable case, that of interacting loops in thermodynamical equilibrium. This can be accomplished by introducing the Boltzmann factor $\exp (-\beta H\{\mathbf{s}\})$ into the Gaussian model (7) (see $\left.{ }^{11,12}\right)$. Then the partition function can be written (we will take here the purely Wiener distribution with $\Lambda$ being the $\delta$ function and with an elementary step equal to $\xi_{0}$ )

$Z=\int D \mathbf{s}(\xi\} \exp \left[-\frac{3}{2 \xi_{0}} \int_{0}^{L}\left(\mathbf{s}^{\prime}(\xi)\right)^{2} d \xi-\beta \frac{\rho_{s} \kappa^{2}}{8 \pi} \oint \oint d \xi_{1} d \xi_{2} \frac{\mathbf{s}^{\prime}\left(\xi_{1}\right) \mathbf{s}^{\prime}\left(\xi_{2}\right)}{\left|\mathbf{s}\left(\xi_{1}\right)-\mathbf{s}\left(\xi_{2}\right)\right|^{3}} d \xi_{1} d \xi_{2}\right]$

Here $\beta=1 / k_{B} T$ is the inverse temperature. A considerable simplification in the evaluation of the partition function can be reached with the use of the Edwards trick (see for details $13,14,15,16$ ), namely, $\exp (-\beta H\{\mathbf{s}\})$ can be written as a the Gaussian path integral over an auxiliary vector field $\mathbf{A}(\mathbf{r})$. After all the transformations, the partition function acquires the form of a Gaussian path integral over the $3 \mathrm{D}$ auxiliary vector field $\mathbf{A}(\mathbf{r})$ and $E=d \ln Z / d \beta$ is (see $\left.{ }^{16}\right)$ :

$$
E=\frac{3}{4 \pi^{2}} \xi_{0} L \rho_{s} \kappa^{2} \int d k \frac{k^{2}}{\left(k^{2}+M^{2}\right)}
$$

with $M^{2}=\beta \xi_{0} L \rho_{s} \kappa^{2} / 2 \mathscr{V}$ ( $\mathscr{V}$ is the volume). For small $k$, the energy spectrum $E(k) \propto\left(k_{B} T\right) k^{2}$, which is nothing but the Rayleigh equipartition law $d E / d^{3} \mathbf{k}=$ const. For large wave numbers $E(k)$ is just about constant. The reason is that for large $k$, the interaction energy in the partition function (9) is smaller and the main contribution appears from the configuration term $\left(\mathbf{s}^{\prime}(\xi)\right)^{2}$, which is related to the connectivity of the line. Hence, we have the same spectrum as for a pure random walk with $H_{D}=2$, discussed in previous Section.

\section{1D Kelvin waves spectrum and 3D velocity spectrum}

In the literature there is discussed the idea of obtaining the $3 \mathrm{D}$ velocity spectrum just by putting it equal to the spectrum of 1D Kelvin waves. For instance, as stated in 17."'We notice that, because the fluctuations of the velocity field are induced by the Kelvin wave fluctuations on the filaments, it is reasonable to expect that

$$
E(k) \sim E_{K W}(k) "
$$

The same idea was used in papers by L'vov et al.(see e.g., 18 ). Details of this activity can be read in a series of papers by L'vov, Nazarenko and coauthors $19,18,20,21$

Let us consider this problem on the basis of general formula (1) (see 16 ). We take $\mathbf{s}(\xi, t)=(x(z, t), y(z, t), z)$ and denote the two-dimensional vector $(x(z, t), y(z, t))$ 
as $a \rho(z, t)$ (where the dimensionless amplitude $a \ll 1$ ). Substituting it into (2) and expanding in powers of $a$, we get,

$$
\begin{aligned}
E= & E_{0}+a^{2} \frac{\rho_{s} \kappa^{2}}{2} \int_{\mathbf{k}} \frac{d^{3} \mathbf{k}}{\mathbf{k}^{2}} \int_{0}^{L} \int_{0}^{L} d z_{1} d z_{2}\left\{\frac{\cos \left(k\left|z_{2}-z_{1}\right|\right)\left(\rho\left(z_{2}\right)-\rho\left(z_{1}\right)\right)^{2}}{2\left|z_{2}-z_{1}\right|^{2}}\right. \\
& \left.-\frac{\sin \left(k\left|z_{2}-z_{1}\right|\right)\left(\rho\left(z_{2}\right)-\rho\left(z_{1}\right)\right)^{2}}{2 k\left|z_{2}-z_{1}\right|^{3}}+\frac{\left(\rho^{\prime}\left(z_{1}\right) \rho^{\prime}\left(z_{2}\right) \sin \left(k\left|z_{2}-z_{1}\right|\right)\right.}{k\left|z_{2}-z_{1}\right|}\right\}
\end{aligned}
$$

The first term $E_{0}$ of the zero-order in amplitude $a$ exactly coincides with the energy spectrum induced by the unperturbed straight line (3), as it should be.

To move further we have to find the correlation characteristics for the fluctuating vector of displacement $\rho\left(z_{2}\right)$. We accept that the ensemble of Kelvin waves has a following power-like spectrum:

$$
\langle\rho(p) \rho(-p)\rangle=A p^{-s} .
$$

We take here the notation $p$ for the one-dimensional vector, conjugated to $z$, reserving the notation $k$ for the absolute value of the wave vector of the 3D field. The formula (14) implies that (see, e.g., $\frac{7}{,}$, Eqs. (4.60),(4.61)) the squared increment for the vector of displacement scales as, $\left\langle\left(\rho\left(z_{2}\right)-\rho\left(z_{1}\right)\right)^{2}\right\rangle \propto\left(z_{2}-z_{1}\right)^{s-1}$. Then the second order correlator $\left\langle\left(\rho^{\prime}\left(z_{2}\right) \rho^{\prime}\left(z_{1}\right)\right)\right\rangle$ scales as $\left\langle\left(\rho^{\prime}\left(z_{2}\right) \rho^{\prime}\left(z_{1}\right)\right)\right\rangle \propto\left(z_{2}-z_{1}\right)^{s-3}$. Substituting it into (14) and counting the powers of quantity $k$, we conclude that the correction $\delta E(k)$ to the spectrum $E(k)$, due to the ensemble of Kelvin waves has a form:

$$
\delta E(k) \propto a^{2} k^{-s+2} .
$$

It is remarkable fact that this quantity coincides formally with the one-dimensional spectrum of $\mathrm{KW} \delta E(p) \propto a^{2} p^{-s+2}$ however this contribution is small, by virtue the smallness of the wave amplitudes $a$, and disappears with the KW.

\section{Conclusions}

Summarizing, it can be concluded that the 3D energy spectrum $E(k)$ consists of several parts. At small $k$, associated with the large scales, on the order of the size of the system, or on the scale of the stirring forcing (grids, propellers, vibrating objects, etc.), the spectrum behaves as $E(k) \propto k^{2}$. We recall again that this is the consequence of the asymptotic behavior $(r \rightarrow \infty)$ of the velocity field, not of thermodynamical equilibrium. for large wave numbers, exceeding the inverse intervortex space $k>2 \pi / \delta$, the energy spectrum $E(k)$ should be close to $k^{-1}$, again regardless of the specific model. The region of intermediate $k$ is the most intriguing and exciting, it depends on fractal properties of lines. Unfortunately, so far there is no theory, predicting the Kolmogorov like spectrum $E(k) \propto k^{-5 / 3}$.

Acknowledgements The work was supported by the grants N 10-08-00369 and N 10-02-00514 from the Russian Foundation of Basic Research, and by the grant from the President Federation on the State Support of Leading Scientific Schools NSh-6686.2012.8. 


\section{References}

1. S. K. Nemirovskii, Phys. Rev. B 57, 5972 (1998).

2. L. Kondaurova and S. K. Nemirovskii, Journal of Low Temperature Physics 138, 555 (2005).

3. C. Nore, M. Abid, and M. E. Brachet, Phys. Rev. Lett. 78, 3896 (1997).

4. C. Nore, M. Abid, and M. E. Brachet, Physics of Fluids 9, 2644 (1997).

5. W. F. Vinen, Phys. Rev. B 61, 1410 (2000).

6. D. S. Boris Nowak, Jan Schole and T. Gasenzer, Phys. Rev. A (2012).

7. U. Frisch, Turbulence (Cambridge University Press, Cambridge, 1995).

8. A. Monin and A. Yaglom, Statistical Fluid Dynamics, Vol. II. (MIT, Cambridge, MA, 1975).

9. S. R. Stalp, L. Skrbek, and R. J. Donnelly, Phys. Rev. Lett. 82, 4831 (1999).

10. S. K. Nemirovskii, M. Tsubota, and T. Araki, Journal of Low Temperature Physics 126, 1535 (2002).

11. S. K. Nemirovskii, Theoretical and Mathematical Physics 141, 1452 (2004).

12. S. Nemirovskii and L. Kondaurova, Journal of Low Temperature Physics 156, 182 (2009), ISSN 0022-2291.

13. H. Kleinert, Gauge Fields in Condenced Matter Physics (World Scientific, Singapore, 1990).

14. S. F. Edwards and M. Warner, Philosophical Magazine A 40, 257 (1979).

15. E. Copeland, D. Haws, S. Holbraad, and R. Rivers, Physica A: Statistical Mechanics and its Applications 179, 507 (1991), ISSN 0378-4371.

16. S. Nemirovskii, submitted to PhysRep, 2012.

17. D. Kivotides, J. C. Vassilicos, D. C. Samuels, and C. F. Barenghi, Phys. Rev. Lett. 86, 3080 (2001)

18. V. Lvov, S. Nazarenko, and O. Rudenko, J. Low Temp. Phys. 153, 140 (2008).

19. V. S. L'vov, S. V. Nazarenko, and O. Rudenko, Phys. Rev. B 76, 024520 (2007).

20. N. Sasa, T. Kano, M. Machida, V. S. L’vov, O. Rudenko, and M. Tsubota, Phys. Rev. B 84, 054525 (2011).

21. L. Boué, R. Dasgupta, J. Laurie, V. L'vov, S. Nazarenko, and I. Procaccia, Phys. Rev. B 84, 064516 (2011). 